\title{
VIBRATION FREQUENCY OF PRESTRESS SLENDER BEAMS RESTING ON WINKLER ELASTIC FOUNDATION
}

\author{
NGUYEN DINH KIEN \\ Institute of Mechanics, VAST
}

\begin{abstract}
The present paper investigates the vibration frequency of slender beams prestressing by axial force and resting on an elastic Winkler foundation by the finite element method. A beam element taking the effects of both the prestress and foundation support into account is formulated using the expression of strain energy. Using the developed element, the natural frequencies of beams having various boundary conditions are computed for different values of the axial force and foundation stiffness. The influence of the axial force and the foundation stiffness on the frequency of the beams is investigated. The effect of partial support by the foundation and the type of mass matrices on the vibration frequency of the beam is also studied and highlighted.
\end{abstract}

\section{INTRODUCTION}

Prestress beams formed by inducing axial force are common structural elements in civil engineering. With the presence of the axial force, both the static and dynamic characteristics of the structure are altered. The effect of the axial load can be explained by alternation of the bending stiffness of the structure under the load. Thus, buckling occurs when the compressive membrane forces are large enough to reduce the bending stiffness to zero [1]. At the same time, the vibration frequency of the beam is very much affected by the membrane force, and it is rapidly reduced with the compressive membrane force.

From practical point of view, the problem of analyzing beams resting on an elastic foundation has many applications. Examples include a rail on a roadbed or a pavement slab on soil. The rail or slab must be analyzed, and the effect of the roadbed or soil support must be modelled. The effect of foundation support on the deflection and buckling of the beam has been studied for different foundation models [2,3]. However, investigation on the effect of membrane force on the vibration characteristics of beams resting on elastic foundation is still hardly found in the literature.

The present paper aims to investigate the vibration frequency of prestress slender beams resting on a Winkler elastic foundation by the finite element method. To this end, a beam element taking the effect of both the prestress and the foundation support into account is formulated. The eigenvalue problem is formed for various types of mass matrices, and then solved for the vibration frequencies. The influence of the axial force, the foundation stiffiness, the type of mass matrix on the vibration frequency of beams having various boundary conditions is investigated in detail.

Following the above introduction, the remainder of this paper is organized as follows: Sec. 2 describes the eigenvalue problem in dynamic analysis of structure by the finite element method. Sec. 3 formulates the element stiffness matrix taking the effect of prestress 


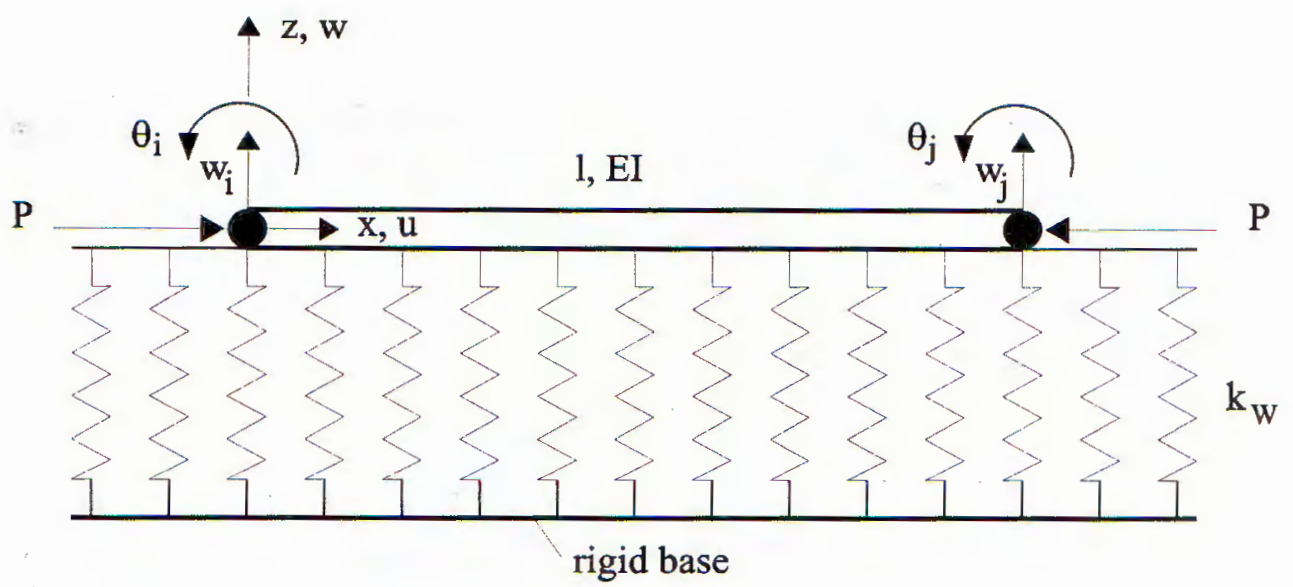

Fig. 1. A two-node beam element on Winkler foundation under axial force

and foundation support into account. Sec. 4 presents various types of mass matrices used in dynamic analysis of beams. The numerical investigation is presented in Sec. 5. The main conclusions of the paper are summarized in Sec. 6.

\section{EIGENVALUE PROBLEM}

Consider an undamped structure. Assuming the structure is discretized into NE number of finite elements. The equation of motion for the discretized structure can be written in the forms $[4,5]$.

$$
\mathbf{M} \ddot{\mathbf{D}}+\mathbf{K D}=\mathbf{F}_{\text {ext }}
$$

where $\mathbf{D}$ is the vector of structural nodal displacements; $\mathbf{M}$ and $\mathbf{K}$ is the structural mass and stiffness matrices, respectively; $\mathbf{F}_{\text {ext }}$ is the vector of nodal external forces; $\ddot{\mathbf{D}}=\frac{d^{2} \mathbf{D}}{d t^{2}}$ is the acceleration of material particles at the structural nodes. The structural mass and stiffness matrices are formed by merging the element mass and stiffness matrices in the standard way of the finite element method.

With no external forces, the structure undergoes harmonic motion (caused, perhaps by initial condition). Thus

$$
\mathbf{D}=\overline{\mathbf{D}} \sin \omega t
$$

where $\overline{\mathbf{D}}$ is the vector of vibration amplitudes of the nodal displacement vector $\mathbf{D}$, and $\omega$ is the circular frequency $(\mathrm{rad} / \mathrm{s})$. Substituting (2.2) into Eq. (2.1) for the case of free vibration, we get

$$
(\mathbf{K}-\lambda \mathbf{M}) \overline{\mathbf{D}}=0
$$

where $\lambda=\omega^{2}$. Eq. (2.3) is called an eigenvalue problem, which gives nontrivial solution when its determinate equals to zero.

Eq. (2.3) can be solved using any standard algorithm to obtain eigenvalues $\lambda$ and their associated eigenvectors. The frequency corresponding the lowest eigenvalue $\lambda$ computed from Eq. (2.3) is called the fundamental frequency [5]. 


\section{PRESTRESS BEAM ELEMENT ON ELASTIC FOUNDATION}

Consider a two-node (denoted $i$ and $j$ ) beam element with length $l$ and flexural rigidity $E I$, prestressed by axial force $P$ as shown in Fig. 1 . The beam is supported by a Winkler elastic foundation, which modelled by linear springs with stiffness represented by $k_{W}$ (unit of force/length ${ }^{2}$ ). In this model, the springs are assumed to be independent each other, and only one parameter $k_{W}$ is represented for the foundation $[2,6]$.

Assuming linear elastic behavior, the strain energy of the element is a contribution from bending strain energy $U_{B}$, energy stored in the foundation $U_{W}$, and potential energy of the axial force $U_{P}[7]$.

$$
U=U_{B}+U_{W}+U_{P}=\frac{1}{2} \int_{0}^{l} E I\left(\frac{\partial^{2} w}{\partial x^{2}}\right) d x+\frac{1}{2} \int_{0}^{l} k_{W} w^{2} d x+\frac{1}{2} \int_{0}^{l} P\left(\frac{\partial w}{\partial x}\right)^{2} d x
$$

where $w$ denotes the transversal displacement of the neutral axis of the beam, and $P$ is positive in tension. In writing Eq. (3.1) we have ignored the effect of axial strain and approximated the shortening of the element as

$$
\Delta=\int_{0}^{l} d u=\int_{0}^{l}\left[\left(1+\left(\frac{\partial w}{\partial x}\right)^{2}\right)^{1 / 2}-1\right] d x \approx \frac{1}{2} \int_{0}^{l}\left(\frac{\partial w}{\partial x}\right)^{2} d x
$$

Following standard approach of the finite element method, we introduce the interpolation scheme for the transversal displacement as

$$
w=\mathbf{N}^{T} \mathbf{w}
$$

where superscript ${ }^{T}$ denotes the transformation of a vector or a matrix; $\mathbf{w}$ is the vector of element nodal displacements with four components

$$
\mathbf{w}=\left\{w_{i} \theta_{i} w_{j} \theta_{j}\right\}^{T}
$$

and $\mathbf{N}$ denotes the matrix of shape functions. With slender beams of the present paper, the Hermitian polynomials for Bernoulli beam element can be adopted as

$$
\mathbf{N}=\left\{\begin{array}{c}
N_{1} \\
N_{2} \\
N_{3} \\
N_{4}
\end{array}\right\}=\left\{\begin{array}{c}
1-3 \frac{x^{2}}{l^{2}}+2 \frac{x^{3}}{l^{3}} \\
x-2 \frac{x^{2}}{l}+\frac{x^{3}}{l^{2}} \\
3 \frac{x^{2}}{l^{2}}-2 \frac{x^{3}}{l^{3}} \\
-\frac{x^{2}}{l}+\frac{x^{3}}{l^{2}}
\end{array}\right\}
$$

From Eqs. (3.3)-(3.5), we can compute

$$
\frac{\partial w}{\partial x}=\mathbf{N}_{x}^{T} \mathbf{w} \quad \text { with } \quad \mathbf{N}_{x}=\left\{\begin{array}{c}
-6 \frac{x}{l^{2}}+6 \frac{x^{2}}{l^{3}} \\
1-4 \frac{x}{l}+3 \frac{x^{2}}{l^{2}} \\
6 \frac{x}{l^{2}}-6 \frac{x^{2}}{l^{3}} \\
-2 \frac{x}{l}+3 \frac{x^{2}}{l^{2}}
\end{array}\right\}
$$


and

$$
\frac{\partial^{2} w}{\partial x^{2}}=\mathbf{N}_{x x}^{T} \mathbf{w} \quad \text { with } \quad \mathbf{N}_{x x}=\left\{\begin{array}{c}
-\frac{6}{l^{2}}+12 \frac{x}{l^{3}} \\
-\frac{4}{l}+6 \frac{x}{l^{2}} \\
\frac{6}{l^{2}}-12 \frac{x}{l^{3}} \\
-\frac{2}{l}+6 \frac{x}{l^{2}}
\end{array}\right\}
$$

Substituting Eqs. (3.3), (3.6) and (3.7) into Eq. (3.1), one gets

$$
\begin{aligned}
U_{B}= & \frac{2}{l^{3}} E I\left[3\left(w_{i}^{2}+w_{j}^{2}\right)+l^{2}\left(\theta_{i}^{2}+\theta_{j}^{2}\right)+3 l\left(w_{i}-w_{j}\right)\left(\theta_{i}+\theta_{j}\right)-6 w_{i} w_{j}+l^{2} \theta_{i} \theta_{j}\right], \\
U_{W}= & \frac{l}{420} k_{W}\left[78\left(w_{i}^{2}+w_{j}^{2}\right)+22 l\left(w_{i} \theta_{i}-w_{j} \theta_{j}\right)+13 l\left(\theta_{i} w_{j}-\theta_{j} w_{i}\right),\right. \\
& \left.+54 w_{i} w_{j}-3 \theta_{i} l^{2} \theta_{j}+2 l^{2}\left(\theta_{i}^{2}+\theta_{j}^{2}\right)\right], \\
U_{P}= & \frac{1}{30 l} P\left[18\left(w_{i}^{2}+w_{j}^{2}\right)+3 l\left(w_{i}-w_{j}\right)\left(\theta_{i}+\theta_{j}\right)-l^{2} \theta_{i} \theta_{j}-36 w_{i} w_{j}+2 l^{2}\left(\theta_{i}^{2}+\theta_{j}^{2}\right)\right] .
\end{aligned}
$$

The element stiffness $\mathbf{k}$ is easily obtained using the symbolic software such as Maple as [8]

$$
\mathbf{k}=\mathbf{k}_{B}+\mathbf{k}_{W}+\mathbf{k}_{P}=\operatorname{hessian}\left(U_{B}+U_{W}+U_{P},\left[w_{i}, \theta_{i}, w_{j}, \theta_{j}\right]\right)
$$

From Eq. (3.8), we obtain

$$
k_{B}=\frac{1}{l^{3}} E I\left[\begin{array}{rrrr}
12 & 6 l & -12 & 6 l \\
6 l & 4 l^{2} & -6 l & 2 l^{2} \\
-12 & -6 l & 12 & -6 l \\
6 l & 2 l^{2} & -6 l & 4 l^{2}
\end{array}\right]
$$

is the standard linear stiffness matrix for a Bernoulli element,

$$
k_{W}=\frac{l}{420} k_{W}\left[\begin{array}{cccc}
156 & 22 l & 54 & -13 l \\
22 l & 4 l^{2} & 13 l & -3 l^{2} \\
54 & 13 l & 156 & -22 l \\
-13 l & -3 l^{2} & -22 l & 4 l^{2}
\end{array}\right]
$$

is the stiffness matrix stemming from the deformation of the Winkler foundation, which having the same form as consistent mass matrix as seen in Subsection 4.1, and

$$
k_{P}=\frac{l}{30} P\left[\begin{array}{rrrr}
36 & 3 l & -36 & 3 l \\
3 l & 4 l^{2} & -3 l & -l^{2} \\
-36 & -3 l & 36 & -3 l \\
3 l & -l^{2} & -3 l & 4 l^{2}
\end{array}\right]
$$

is the matrix stemming from the effect of axial force. Thus, the linear stiffness matrix of Bernoulli beam element is augmented by the foundation stiffness matrix and the stress stiffness matrix stemming from the axial force $P$. All the derived stiffness matrices are symmetric.

\section{ELEMENT MASS MATRICES}

A mass matrix is a discrete representation of a continuous distribution of mass. In the present work, the elastic foundation is considered massless as usually assumed in analysis 
of beams on foundation $[9,10]$. Thus, the element mass matrix is contributed from the mass of the beam only.

There are different ways of deriving a mass matrix for a beam element. This section briefly summarizes some types of mass matrices often employed in dynamic analysis of structures, which will be employed in Section 5 in numerical investigation. The detail discussion on the mass matrices is given in $[5,11]$.

\subsection{Consistent mass matrix}

A consistent mass matrix is obtained by using the same shape functions as used in generating the element stiffness matrix, and defined by

$$
\mathbf{m}=\int_{V_{e}} \rho \mathbf{N}^{T} \mathbf{N} d V
$$

where $\mathbf{N}$ is defined by Eq. (3.5) and $V_{e}$ is the element volume. For a uniform beam element, we can easily compute the element mass matrix $\mathbf{m}$ by substituting Eq. (3.5) into (4.1), and obtain

$$
\mathbf{m}=\frac{m}{420}\left[\begin{array}{cccc}
156 & 22 l & 54 & -13 l \\
22 l & 4 l^{2} & 13 l & -3 l^{2} \\
54 & 13 l & 156 & -22 l \\
-13 l & -3 l^{2} & -22 l & 4 l^{2}
\end{array}\right]
$$

where $m=\rho l A$ ( $\rho$ is mass density, and $A$ is the cross-sectional area) is the total element mass.

\subsection{Lumped mass matrix}

A lumped mass matrix is simply obtained by placing the partial masses $m_{i}$ at node $i$ of the element such as $\sum m_{i}$ is the total element mass. For a beam element, particle lump often has rotary inertia and the mass matrix has the following form [11]

$$
\mathbf{m}=\frac{m}{2}\left[\begin{array}{cccc}
1 & 0 & 0 & 0 \\
0 & \alpha l^{2} / 210 & 0 & 0 \\
0 & 0 & 1 & 0 \\
0 & 0 & 0 & \alpha l^{2} / 210
\end{array}\right]
$$

where $m_{22}$ and $m_{44}$ are the coefficients accounted for the rotary inertia, and often selected as mass moment of inertia of a uniform slender bar of length $l / 2$ and mass $m / 2=\rho l A / 2$ spinning about one end, so that $\alpha=17.5$.

\subsection{HRZ lumping mass matrix}

The HRZ lumping mass matrix is an effective method for producing a diagonal mass matrix. The method is proposed by Hilton, Rock and Zienkiewicz [12].The central idea of the method is used only the diagonal terms of the consistent mass matrix, but to scale them in such a way that the total mass of the element is preserved. The method of deriving is given detail in [12], and the mass matrix has following form

$$
\mathbf{m}=\frac{m}{78}\left[\begin{array}{cccc}
39 & 0 & 0 & 0 \\
0 & l^{2} & 0 & 0 \\
0 & 0 & 39 & 0 \\
0 & 0 & 0 & l^{2}
\end{array}\right]
$$




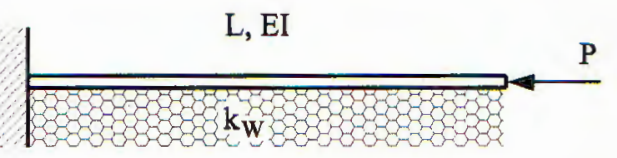

(a)

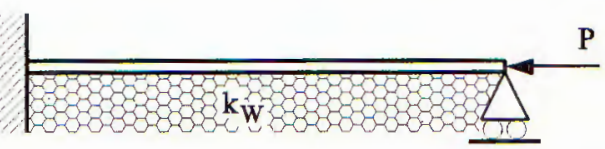

(c)

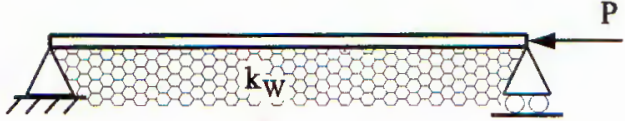

(b)

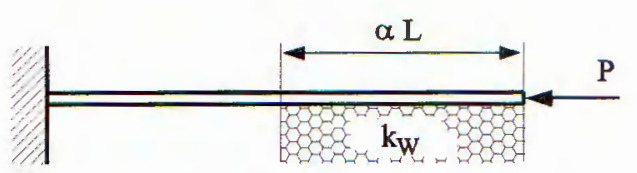

(d)

Fig. 2. Beams with various boundary conditions on elastic foundations for numerical investigation

All the mass matrices presented in this Section will be employed in the numerical investigation in Section 5, and their accuracy will be discussed in consequence.

\section{NUMERICAL INVESTIGATION}

The eigenvalue problem stated by Eq. (2.3) is formed from the finite element formulations developed in Sections 3 and 4 , then solved for the frequencies of prestressed beams shown in Fig. 2. Various boundary conditions are considered: clamped at one end and free at other (denoted CF, Fig. 2a), simply supported (SS, Fig. 2b), clamped at one end and simply supported at other (CS, Fig. 2c). The effect of partially supported as shown in Fig. 2d for CF beam, where $\alpha L(0 \leq \alpha \leq 1)$ denoted the supporting part, is also investigated. The geometry and material data for the beams are as follows:

$$
L=5 \mathrm{~m} ; \quad A=0.01 \mathrm{~m}^{2}, \quad I=1 \times 10^{-5} \mathrm{~m}^{4} ; \quad E=2.1 \times 10^{11} \mathrm{~N} / \mathrm{m}^{2}, \quad \rho=7860 \mathrm{~kg} / \mathrm{m}^{3},
$$

where $L, A, I, E$ and $\rho$ denote the total length, cross-sectional area, second moment of inertia of cross-section, elastic modulus and mass density of the beams, respectively.

\subsection{Unsupported beams}

This Subsection aims to compute the vibration frequencies of the beams without foundation support under the axial force. To this end, the foundation stiffness parameter $k_{W}$ is set to be zero, and the variation of the frequency with the axial force is investigated. For the simplicity of discussion, we introduce the so-called frequency parameter $\gamma$, defined as

$$
\gamma=\frac{\rho A L^{4}}{E I} \omega_{1}^{2}
$$

where $\omega_{1}$ denotes the fundamental frequency of the beams. In addition, a non-dimensional loading parameter $\mu$ determined amplitude of the axial force is also introduced as

$$
\mu=\frac{L^{2}}{E I} P
$$

The numerical results reported below have been obtained by using a mesh of 5 -equal elements.

Table 1 lists the frequency parameter $\gamma$ of unsupported SS beam computed at various values of loading parameter $\mu$ and different mass matrix types. The corresponding values 
Table 1. Frequency parameter $\gamma$ of unsupported SS beam at various values of compressive axial force and different mass matrices ( 5 - equal elements)

\begin{tabular}{crrrrr}
\hline$\mu$ & 0 & \multicolumn{1}{c}{-2} & \multicolumn{1}{c}{-4} & \multicolumn{1}{c}{-6} & -8 \\
\hline Consistent & 97.4299 & 77.6907 & 57.9514 & 38.2122 & 18.4730 \\
error (\%) & 0.0214 & 0.0268 & 0.0359 & 0.0543 & 0.1122 \\
\hline Lumped & 94.2862 & 75.1839 & 56.0816 & 36.9793 & 17.8769 \\
error (\%) & -3.2059 & -3.2007 & -3.1919 & -3.1740 & -3.1180 \\
\hline HRZ lumping & 96.4114 & 76.8787 & 57.3458 & 37.8129 & 18.2800 \\
error (\%) & -1.0242 & -1.0187 & -1.0095 & -0.9911 & -0.9337 \\
\hline
\end{tabular}

Table 2. Frequency parameter $\gamma$ of unsupported CF beam at various values of compressive axial force and different mass matrices ( 5 equal elements)

\begin{tabular}{lcrrrr}
\hline \multicolumn{1}{c}{$\mu$} & 0 & -0.5 & -1.0 & -1.5 & -2.0 \\
\hline Consistent & 12.3627 & 10.0067 & 7.5826 & 5.0829 & 2.4994 \\
Lumped & 11.7439 & 9.4879 & 7.1747 & 4.7988 & 2.3540 \\
HRZ lumping & 11.8659 & 9.5889 & 7.2531 & 4.8527 & 2.3813 \\
\hline
\end{tabular}

Table 3. Frequency parameter $\gamma$ of unsupported CS beam at various values of compressive axial force and different mass matrices (5 - equal elements)

\begin{tabular}{lccccc}
\hline \multicolumn{1}{c}{$\mu$} & 0 & -5 & -10 & -15 & -20 \\
\hline Consistent & 237.8450 & 180.0220 & 121.6148 & 62.5015 & 2.5192 \\
Lumped & 228.8115 & 173.1246 & 116.9066 & 60.0514 & 2.4189 \\
HRZ lumping & 234.7931 & 177.6980 & 120.0323 & 61.6797 & 2.4856 \\
\hline
\end{tabular}

for $\mathrm{CF}$ and $\mathrm{CS}$ beams are given in Tables 2 and 3, respectively. The results listed in Tables 1-3 have been obtained with a mesh of 5 -equal elements. The percentage error displayed in Table 1 is defined as

$$
\operatorname{error}(\%)=\frac{\gamma_{\text {compute }}-\gamma_{\text {exact }}}{\gamma_{\text {exact }}} \times 100 \%
$$

where $\gamma_{\text {exact }}$ is obtained from the exact fundamental frequency reported in [13] for the case of simply supported beam as

$$
\omega_{\text {exact }}^{2}=\frac{\pi^{2} E I}{\rho A L^{4}}\left(1+\frac{L^{2}}{E I} P\right), \quad \text { so that } \quad \gamma_{\text {exact }}=\pi^{4}\left(\frac{\mu}{\pi^{2}}+1\right)
$$

It is noted that, to the author knowledge, the exact solution for other cases of the boundary conditions is not available in the literature, so that the computed frequencies listed in Tables 2 and 3 may be useful for practice.

It is seen from Table 1 the consistent mass matrix gives the most accurate fundamental frequency, and the computed frequency using the consistent mass matrix is always higher than the exact value. The frequencies computed by both the lumped and HRZ lumping matrices are lower than the exact values as expected [14]. The frequency of the'beams is lower for a higher value of the compressive axial force, and it becomes zero at the buckling Euler load, regardless of the boundary conditions and the type of mass matrices (see also Tables 2 and 3). The buckling Euler loads for SS, CF and CS beams are given in [15], and 
Table 4. Frequency parameter $\gamma$ of unsupported SS beam computed by different element numbers and with $\mu=-4$

\begin{tabular}{ccrrrr}
\hline & \multicolumn{5}{c}{ element number } \\
Mass matrices & 2 & 4 & \multicolumn{1}{c}{6} & \multicolumn{1}{c}{8} & \multicolumn{1}{c}{10} \\
\hline Consistent & 58.6862 & 57.9810 & 57.9407 & 57.9339 & 57.9320 \\
error (\%) & 1.3041 & 0.0868 & 0.0174 & 0.0055 & 0.0023 \\
\hline Lumped & 47.9114 & 55.0888 & 56.6348 & 57.1950 & 57.4579 \\
error (\%) & -17.2952 & -4.9056 & -2.2369 & -1.2698 & -0.8162 \\
\hline HRZ lumping & 54.2422 & 57.0178 & 57.5243 & 57.7019 & 57.7842 \\
error (\%) & -6.3670 & -1.5759 & -0.7016 & -0.3949 & -0.2529 \\
\hline
\end{tabular}

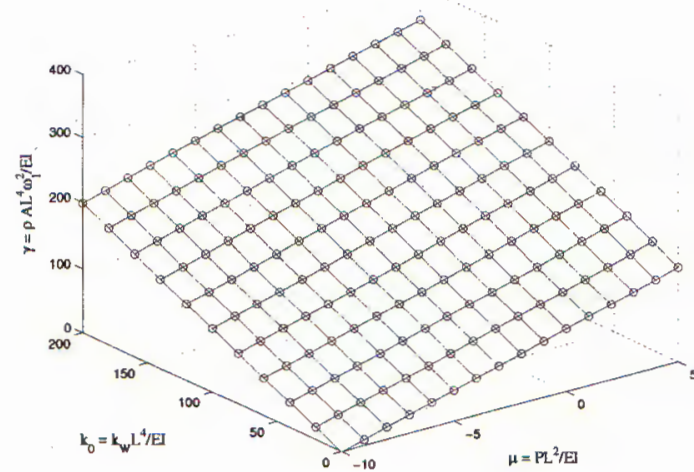

Fig. 3. Influence of axial force and foundation stiffness on frequency parameter of SS beam

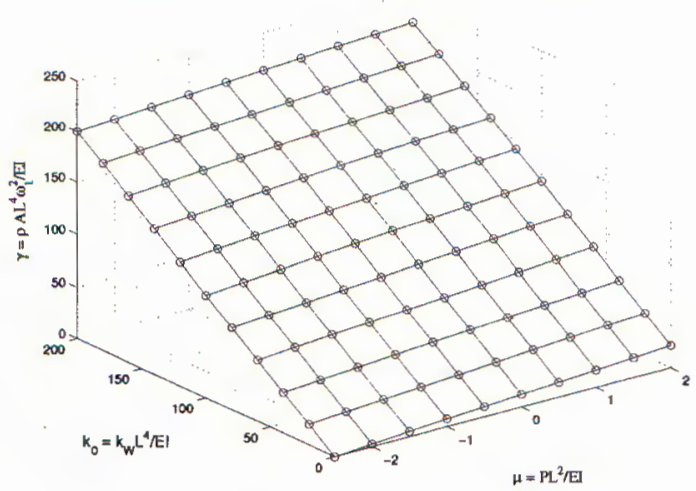

Fig. 4. Influence of axial force and foundation stiffness on frequency parameter of CF beam

in the term of loading parameter $\mu$ are written by

$$
\mu_{\mathrm{SS}}^{\mathrm{E}}=-\pi^{2}, \quad \mu_{\mathrm{CF}}^{\mathrm{E}}=-\frac{\pi^{2}}{4}, \quad \mu_{\mathrm{CS}}^{\mathrm{E}}=-2.046 \pi^{2}
$$

where the superscript ${ }^{E}$ stands for 'Euler'. The HRZ lumping mass matrix is much more accurate comparing the lumped mass matrix, and the error using the HRZ matrix may be acceptable in regarding its simple form (only nonzero diagonal coefficients). To demonstrate the convergency of the method, the computation has been performed for SS beam using various numbers of elements, and the results are listed in Table 4." Is is clearly seen from Table 4, the frequency of the beam rapidly converges towards the exact value, regardless of the mass matrix type. The consistent mass matrix shows a superiority in the convergency comparing its counterparts, but the HRZ lumping matrix is also a very good one.

\subsection{Fully supported beams}

The aim of this Subsection is to investigate the effects of combined prestress and foundation support on the frequency of the beams. The beams are fully supported by the foundation as shown in Fig. 2a-c. For the sake of simplicity, we introduce the dimensionless 
parameter $k_{0}$ represented the foundation stiffness as

$$
k_{0}=\frac{L^{4}}{E I} k_{W}
$$

The results reported below are computed with a mesh of 10 equal elements and only the consistent mass matrix is employed.

Fig. 3 shows the influence of prestress and foundation stiffness on the frequency parameter of fully supported SS beam. The corresponding figures for fully supported $\mathrm{CF}$ and CS beams are shown in Fig. 4 and Fig. 5, respectively. As clearly seen from the figures, at a given value of the axial force the frequency parameter of the beams is linear increased with an increment in the foundation stiffness, regardless of the boundary conditions. For all the beams, the frequency is lower at a higher value of the compressive axial load $(P<0)$, and higher for a higher value of the tensile axial force $(P>0)$. In other words, the foundation and tensile axial force increases the vibration frequency of beams resting on elastic foundation, regardless of the boundary conditions.

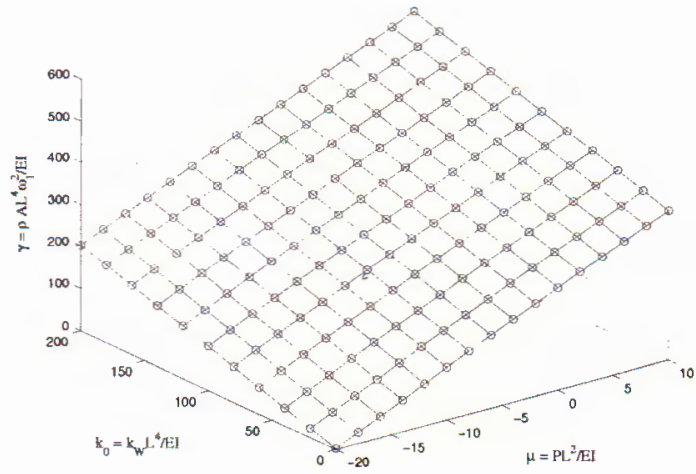

Fig. 5. Influence of axial force and foundation stiffness on frequency parameter of CS beam

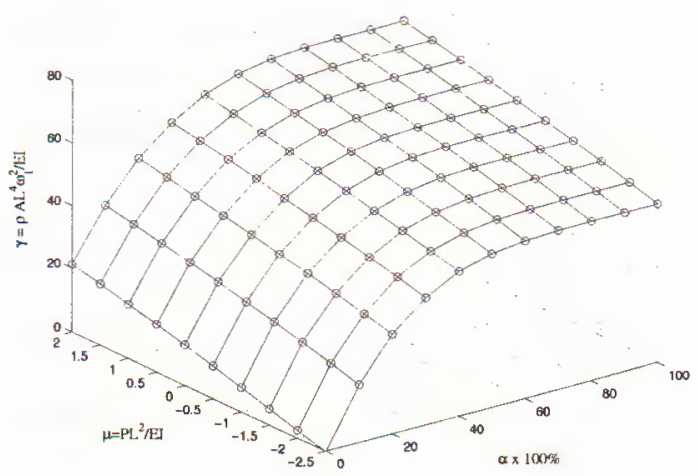

Fig. 6. Frequency parameter of partially supported $\mathrm{CF}$ beam at various values of axial force and supported percentages $\left(k_{0}=50\right)$

\subsection{Partially supported beams}

The beam in this Subsection is assumed to be partially supported by the elastic foundation, and only CF beam as shown in Fig. 2d is considered herewith. The computation is performed for the foundation stiffness parameter $k_{0}=50$, using a mesh of 10-equal elements and with the consistent mass matrix only.

Fig. 6 shows the computed frequency of CF beam partially supported by the elastic foundation at various supporting percentages $(\alpha \times 100 \%)$ and at different values of the axial force. The figure shows a nonlinear change in the frequency of the beam with the supporting percentage. For supporting percentage less than $60 \%$, the frequency rapidly increases with an increment in the supporting percentage, it then increases very slowly. The frequency changes very little when the supporting percentage is higher than $70 \%$. 


\section{CONCLUSIONS}

The paper has investigated the effects of prestress by axial force and foundation stiffness on the vibration frequency of beams resting on a Winkler elastic foundation by the finite element method. A beam element taking the effect of both the prestress and foundation support into account has been formulated using the expression of strain energy. The eigenvalue problem was solved to obtain the natural frequencies of beams having various boundary conditions. The accuracy of different mass matrices in determining the vibration frequency of the beams has also been investigated. The main conclusions obtained from the numerical investigations can be summarized as follows:

- The vibration frequency of the beams is lower at a higher value of compressive force, but higher at a higher tensile force, regardless of the boundary conditions and the type of the mass matrices.

- The vibration frequency linearly depends on the foundation stiffness and the axial force.

- The vibration frequency nonlinearly depends on the supporting percentage. The increment in the frequency is rapid at low supporting percentages, and very slow at high supporting percentages.

- The consistent mass matrix is the most accurate in computing the vibration frequency of the beams, but the HRZ lumping mass matrix is also very good in consideration of its diagonal form.

Acknowledgement. The present work has been financed in part by the National Program on Fundamental Research.

\section{REFERENCES}

1. A. Ghali and A. M. Neville, Structural Analysis. A Unified Classical and Matrix Approach, E \& FN Spon, London, Third edition, 1995.

2. M. Hetényi, Beams on Elastic Foundation, The University of Michigan Press, Ann Arbor, 1946.

3. S. P. Timoshenko and J. M. Gere, Theory of Elastic Stability, McGraw-Hill, New York, Second edition, 1961.

4. M. Géradin and R. Rixen, Mechanical Vibrations. Theory and Application to Structural Dynamics, John Wiley and Sons, Chichester, Second, 1997.

5. L. Meirovithch, Fundamentals of Vibrations, McGraw-Hill, Boston, 2001.

6. S. C. Dutta and R. Roy, A critical review on idealization and modeling for interaction among soil-foundation-structure system, Computers \& Structures 80 (2002) 15791594.

7. M. S. El Naschie, Stress, Stability and Chaos in Structural Engineering: an Energy Approach, McGraw-Hill, London, 1990.

8. Maple V, Language Reference Manual, Spring-Verlag, New York, 1991.

9. T. Yokoyama, Vibration analysis of Timoshenko beam-column on two-parameter elastic foundation, Computers \& Structures 61 (1996) 995-1007.

10. G. V. Rao, Large-amplitude free vibrations of uniform beams on Pasternak foundation, Journal of Sound and Vibration 263 (2003) 954-960.

11. T. J. R. Hughes, The Finite Element Method. Linear Static and Dynamic Finite Element Analysis, Dover publication, Inc., Mineola, 2000. 
12. E. Hilton, T. Rock, O. C. Zienkiewicz, A note on mass lumping and related process in the finite element method, Earthquake Engineering and Structural Dynamics 4 (1976) 245-249.

13. J. S. Przemieniecki, Theory of Matrix Structural Analysis, Dover Publications Inc., New York, 1985.

14. R. D. Cook, D. S. Malkus and M. E. Plesha, Concepts and Applications of Finite Element Analysis, John Wiley \& Sons, New York, Third edition 1989.

15. J. M. Gere and S.P. Timoshenko, Mechanics of materials, Chapman \& Hall, London, Third SI edition, 1995.

Received July 12, 2005

Revised August 20, 2005

\section{TẦN SỐ DAO DỘNG CƯA DẦM MẢNH DỤ ỨNG LỰC NẰM TRÊN NỀN DÀN HỒI WINKLER}

Bài báo nghiên cứu tần số dao động của dầm mảnh dữ ứng lực do lực dọc trục, nằm trên nền đàn hồi Winkler bằng phương pháp phần tử hựu hạn. Phần từ dầm tính tới ảnh hưởng của dự ứng lực và nền đàn hồi được xây dựng từ biểu thức năng lượng biến dạng. Tần số dao động của dầm có các điều kiện biên khác nhau với các giá trị khác nhau của lực dọc trục và độ cứng nền được xác định trên cơ sở phần tử xây dựng. Sự phụ thuộc của tần sổ dao dộng vào giá trị lực dọc trục và độ cứng nền được khảo sát chi tiểt. Ảnh hương của các loại ma trận khối lượng khác nhau và tham số xác định phần nền dầm tựa nên cũng được nghiên cứu. 